\title{
Periodontal Tissue Formation by Reaggregation System in Mice
}

\author{
Hitoshi Yamamoto ${ }^{1,2)}$, Jinglei Cai ${ }^{3)}$, Sung-Won Cho ${ }^{3)}$, Jae-Young Kim ${ }^{4)}$ and Han-Sung Jung ${ }^{3)}$ \\ 1) Department of Histology, Cytology and Developmental Anatomy, Nihon University School of Dentistry at Matsudo, 2-870-1, Sakaecho \\ Nishi, Matsudo City, Chiba 270-8587, Japan \\ 2) Research Institute of Oral Science, Nihon University School of Dentistry at Matsudo, 2-870-1, Sakaecho Nishi, Matsudo City, Chiba \\ 270-8587, Japan \\ 3) Division in Anatomy and Developmental Biology, Department of Oral Biology, College of Dentistry, Yonsei University, 134 Shinchon \\ Dong, Seodaemun-Gu, Seoul 120-752, Korea \\ 4) Department of Biochemistry, School of Dentistry, Kyungpook National University, 101, Dongin-Dong, 2-Ga, Joong-Gu, Daegu \\ 700-422, Korea
}

(Accepted for publication, May 18, 2009)

\begin{abstract}
The reaggregation system is a special technique developed to clarify the essential characteristics of the mesenchyme. In this study, the reaggregation system was performed using mouse lower first molar at E13.5 and the reaggregated tooth germs were transplanted into the kidney capsule for 6 weeks to investigate tooth root formation. The reaggregated tooth was surrounded by bone. The reaggregated tooth germ produced a single thick root like a taurodontium (cuneiform tooth), although mouse lower first molar has two roots in vivo. Histologically, the reaggregated mesenchyme could form not only tooth elements including enamel, dentin, dental pulp and cementum, but also periodontal tissue including periodontal ligament-like structure and alveolar bone-like structure. The periodontal ligament-like structure has a regular width between the cementum and alveolar bone-like structure. Moreover, Sharpy's fiber-like structure was inserted into the cementum and alveolar bone-like structure. In conclusion, the reaggregated mesenchyme has the ability to form both tooth and periodontal tissue without information from the epithelium. In addition, this reaggregation system may be a useful method for regenerative dentistry, since tooth and periodontal tissue can be produced en bloc.
\end{abstract}

Key words: Periondontium, Reaggregation system, Molar, Mouse

\section{Introduction}

It is well known that tooth morphogenesis is an excellent model system for epithelial-mesenchymal interactions, which induce the initiation and development of tooth germs ${ }^{1,2)}$. These reciprocal tissue interactions lead to the position of individual tooth germ, cuspal patterning, cell differentiation and synthesis of mineralized matrices $^{3)}$. In mouse first molar, the oral epithelium derived from ectoderm thickens in the putative tooth forming area on embryonic day 11 (E11). Next, the epithelium invaginates into the underlying mesenchyme derived from neural crest and forms the tooth bud on E12.5. At this stage, mesenchymal cells condense around the tooth bud. Then, the epithelial area of tooth germ, enamel organ, folds and creates a cap-like structure on E14. The mesenchymal cells condense at a hollow place under the enamel organ resulting in dental papilla. Moreover, enamel organ and dental papilla are covered by fibrous-rich mesenchyme, dental follicle, at this stage.

Correspondence to: Hitoshi Yamamoto, DDS, PhD.Department of Histology, Cytology and Developmental Anatomy, Nihon University School of Dentistry at Matsudo, 2-870-1, Sakaecho Nishi, Matsudo City, Chiba 270-8587, Japan. E-mail: yamamoto.hitoshi@nihon-u.ac.jp
Previous recombinant experiments have demonstrated that the epithelium could induce tooth formation prior to the bud stage. However, the potential for tooth formation shifted from the epithelium to the mesenchyme at the early bud stage. At the late bud stage, the mesenchyme already takes the initiative for tooth formation against the epithelium ${ }^{4-6)}$.

The reaggregation system has previously already been used to examine a feather bud, a limb bud ${ }^{7)}$ and a tooth ${ }^{8)}$. Using the reaggregation system, the essential characterization of the mesenchyme cells as well as the effects of the epithelialmesenchymal interaction can be examined. Previous reaggregation experiment using E13.5 mouse lower first molar tooth germ was demonstrated that the dental mesenchyme has the ability to produce a tooth crown, although the mesenchyme loses information from the dental epithelium as an essential characteristic. In addition, the newly formed tooth has an enamel structure similar to that of normal tooth in vivo ${ }^{8)}$. Since previous study only investigated formation up to 14 days after reaggregation, there is no information about tooth root formation of reaggregated tooth germs.

This study adopted a reaggregation system to investigate dental 
mesenchymal cells as in the previous study ${ }^{8}$. After reaggregation experiment, reaggregated tooth germs were transplanted into kidney capsule for 6 weeks. Scanning electron microscope (SEM) observation and light microscopy (LM) were examined to investigate reaggregated tooth root formation. The results show that the reaggregated tooth formed a tooth root. Moreover, periodontal tissue, namely, cementum, periodontal ligament-like structure and alveolar bone-like structure, were formed en bloc. These findings suggest that this reaggregation system may be a useful method for regenerative and developmental research.

\section{Materials and Methods}

Animal Care and Use Committee of Nihon University School of Dentistry at Matsudo approved procedures used in this study (No. 03-0018).

\section{Animals}

Twenty-four ICR mice (Mus musculs) on embryonic day 13.5 (E13.5) (vaginal plug=E0) were examined.

\section{Reaggregation system and kidney transplantation}

The reaggregation system was described previously ${ }^{8}$. Briefly, the first molar tooth germs from the lower jaw were dissected on E13.5 under a stereoscopic microscope in phosphate buffered saline (PBS). The germs were incubated at room temperature in $1.2 \mathrm{U} / \mathrm{ml}$ dispase II (Roche Diagnostics, Germany) for $12.5 \mathrm{~min}$ and washed in Dulbecco's minimum essential medium (Bio Whittaker, USA) containing 10\% fetal bovine serum (GIBCO, USA). After separation of the epithelium and the mesenchyme, the epithelium remained intact in the media. The mesenchyme was triturated into single cells by drawing them through pipettes. The dissociated cells were repelleted by centrifugation $(6,500 \mathrm{rpm}$ for $4 \mathrm{~min}$ ) and were allowed to reaggregate for $1 \mathrm{~h}$ at $37^{\circ} \mathrm{C}$. Then, the epithelium was combined with the reaggregated mesenchyme for 1 day at $37^{\circ} \mathrm{C}$. After 1 day incubation, the reaggregated tooth germs were transplanted into kidney capsule for 6 weeks using male adult mice as hosts.

\section{Tissue preparation}

The host mice were perfused with $4 \%$ paraformaldehyde in $0.1 \mathrm{M}$ phosphate buffer ( $\mathrm{pH}$ 7.4) for SEM and LM. For SEM observation, the reaggregated tooth including bone was dissected from the kidney. In some specimens, bone was detached from the reaggregated tooth. After taking a photo using a stereoscopic microscope (SZX12, OLYMPUS, Japan), the specimens were embedded in polyester resin (No. 105, Marumoto Struers, Japan) and were polished. The polishing surface was etched by $0.5 \%$ hydrochloride solution for 30 seconds. After critical point drying and coating with platinum, specimens were observed by SEM (S2700, Hitachi, Japan). For light microscopy, reaggregated tooth including bone between the kidney capsule and cortex were

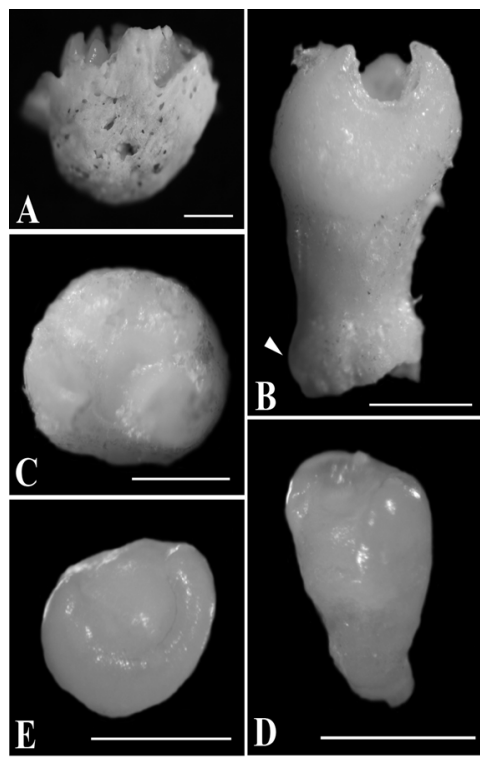

Figure 1 (A) Bone surrounding the reaggregated tooth. The reaggregated tooth is surrounded by bone except for occlusal portion of the tooth crown. (B) Side view of the reaggregated tooth. The reaggregated tooth has a single thick root. At the apical portion of the root, the cementum develops well (arrowhead). (C) Occlusal view of the reaggregated tooth. The shape of the reaggregated tooth differs from that of normal lower first molar. (D) Side view of the second reaggregated tooth. (E) Occlusal view of the second reaggregated tooth. The second reaggregated tooth looks like a dwarfed tooth. bars: $500 \mu \mathrm{m}$

dissected out and fixed with $4 \%$ paraformaldehyde in $0.1 \mathrm{M}$ phosphate buffer ( $\mathrm{pH} 7.4)$ at $4{ }^{\circ} \mathrm{C}$ overnight. The specimens were embedded in paraffin by conventional methods. Sections $5 \mu \mathrm{m}$ thick were mounted on APS-coated slides, then stained with hematoxylin-eosin (H-E).

\section{Results}

\section{Stereoscopic microscope observation}

To clarify the state of the reaggregated tooth in the kidney capsule 6 weeks after transplantation, the stereoscopic microscope observation was performed. Since bone surrounded the reaggregated tooth, the occlusal area of tooth crown was observed on side view (Fig. 1-A). The reaggregated tooth had a completed crown and a single thick root (Fig. 1-B). Moreover, the apical part of root was thick (Fig. 1-B arrowhead). On occlusal view, the shape of tooth crown was not similar to normal lower first molar (Fig. 1-C). Occasionally, another reaggregated tooth (second reaggregated tooth) was found by the reaggregation system (Fig. 1-D, E). This second reaggregated tooth looked like a dwarfed tooth, since the size was very small and the shape was totally different from that of either lower first or second molar in vivo (Fig. 1-D, E).

\section{SEM observation}


Hitoshi Yamamoto et al.: Periodontium by Reaggregation System

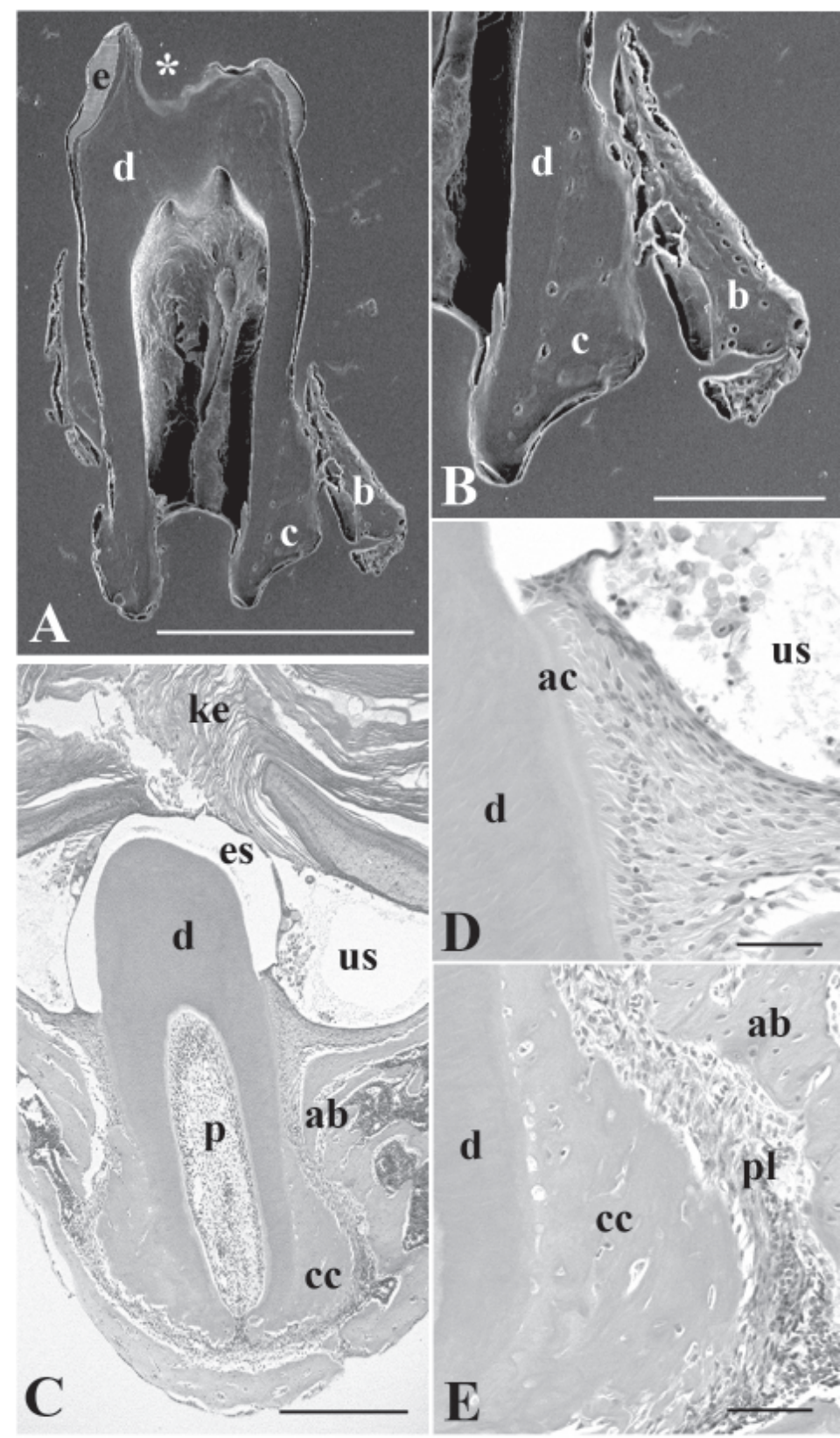

Figure 2. (A) SEM image of the reaggregated tooth with bone. (B) Higher magnification of the apical part of (A). Bone (b) exists at the middle and apical part of the reaggregated tooth. However, cementum (c) is thick at the apical part of the reaggregated tooth, although cementum is thin at the middle part of the reaggregated tooth. (C) H-E section of whole reaggregated tissue. (D) Higher magnification of the cervical portion of (C). (E) Higher magnification of the apical portion of (C). The reaggregated tissue includes thick keratinized epithelium (ke), enamel (enamel space: es), dentin (d), acellular (ac) and cellular cementum (cc), dental pulp (p), periodontal ligament-like structure ( $\mathrm{pl})$ and alveolar bone-like structure (ab). Sharpy's fiber-like structure inserts into both the acellular and cellular cementum and alveolar bone-like structure. The collagen bundles did not run regularly and alveolar bone-like structure appears similar to woven bone. us: unidentified space, bars: (A) $333 \mu \mathrm{m}$, (B) $150 \mu \mathrm{m}$ (C) $250 \mu \mathrm{m}$, (D) $20 \mu \mathrm{m}$, (E) $50 \mu \mathrm{m}$
To analyze the three-dimensional structure of the reaggregated tooth surrounded by bone, ground surface was examined by SEM. The reaggregated tooth had an enamel-free area similar to that of normal mouse molar (Fig. 2-A). The enamel structure was divided into three layers based on the running patterns of enamel prisms like a normal molar (data not shown). At the apical portion of reaggregated tooth root, thick cellular cementum was observed between dentin and bone (Fig. 2-B). However, cellular cementum was not detected between dentin and bone at the middle part of tooth root (Fig. 2-A).

\section{LM observation using $\boldsymbol{H}$-E staining}

To observe the fine structure of the reaggregated tooth, sections were stained by H-E. Between the kidney capsule and cortex, thick keratinized epithelium, enamel space, dentin, pulp, cementum (acellular and cellular), periodontal ligament-like structure and alveolar bone-like structures were observed (Fig. 2-C). Alveolar bone-like structure was comprised of woven bone and completely surrounded the reaggregated tooth. Bundles of collagen fibers located in the periodontal ligament-like structure were inserted into cementum (acellular and cellular) and alveolar bone-like structure. However, collagen bundles did not run regularly in periodontal ligament-like structure. Next to the enamel space, there was an unidentified space that is not found in normal tooth development (Fig. 2-C). Some cells were detected in this unidentified space (Fig. 2-D). Between cementum and alveolar 
bone-like structure, periodontal ligament-like structure showed a constant width. However, in some sections, the width of periodontal ligament is very narrow at the apical portion of the reaggregated tooth (data not shown), although there was no ankylosis between the cementum and alveolar bone-like structure in any sections. There was active bone marrow including megakaryocytes and precursor cells of leukocyte (data not shown) in the alveolar bone-like structure (Fig. 2-C).

\section{Discussion}

The reaggregation system is a useful method of investigating the essential properties of mesenchyme ${ }^{7,8)}$. Previous reaggregation experiment using E13.5 mouse lower first molar demonstrated that the dental mesenchyme has an ability to produce the tooth as an essential property, although even dental mesenchyme loses information from the dental epithelium ${ }^{8)}$. However, since previous study investigated only the newly formed tooth crown formation process, there is no information about the root formation process of the reaggregated tooth. Therefore, reaggregated tooth germs were incubated in kidney capsules for 6 weeks to investigate reaggregated tooth root formation. As a result, the reaggregated tooth could form a root that included acellular and cellular cementum, although the reaggregated tooth had only a single thick root. Moreover, periodontal ligament-like structure and alveolar bone-like structures were observed with reaggregated tooth. In other words, tooth and periodontal tissue could be produced en bloc by the reaggregation system. In addition, Nakao et al. ${ }^{9}$ described that the transplanted tooth germ into the subrenal capsule using incisor tooth germ in mice was surrounded by alveolar bonelike structure and periodontal ligament-like structure. These findings suggest that reaggeregated mesenchymal cells can differentiate into cementoblasts, fibroblasts and osteoblasts respectively similar to normal periodontium development from a dental follicle, although reaggregated mesenchymal cells lose the signals including positional information from the dental epithelium. In order for this method to be used for regenerative treatment in the future, remaining problems must be solved. For example, the alveolar bone-like structure was woven bone and collagen bundles did not run regularly in the periodontal ligamentlike structure, since the reaggregated tooth grew in the kidney capsule without mechanical stress. In clinical dentistry, however, it is considered that regeneration of tooth and periodontal tissue "en bloc" would be a better method than the "individual" regeneration method of tooth and periodontal tissue respectively. Therefore, this reaggregation system may be a useful method for both regenerative and developmental research and clinical dentistry, since tooth and periodontal tissue can form en bloc.

The number and shape of the tooth root were different from that of normal root. Reaggregated tooth has a single thick root like taurodontism (cuneiform tooth) ${ }^{10)}$. It was considered that taurodontism may be caused by a failure of the invagination of Hertwig's epithelial root sheath in a horizontal direction ${ }^{11)}$. Moreover, it was reported that taurodontism is involved in ectodermal abnormality ${ }^{12}$. According to these reports, a new epithelial-mesenchymal interaction may be acquired by the reaggregation system. Moreover, recently it is reported that transplanted tooth using normal tooth germ of mandibular first molar in mice has only one tooth root, although cementum distribution pattern is similar to normal molar in vivo ${ }^{13)}$ These results suggest that the microenvironment surrounding tooth germ may be an important factor for multi-root formation.

In the present study, a second reaggregated tooth was occasionally found in the kidney capsule, although a third reaggregated tooth was never observed. This second reaggregated tooth was similar to dwarfed tooth, since the size and shape were completely different from those of normal first and/or second lower tooth. Since the shape of the crown was modulated by the number of mesenchymal cells ${ }^{14)}$, the formation of the second reaggregated tooth may depend on the ratios of cell number in the epithelium and reaggregated mesenchyme.

\section{Acknowledgements}

This study was supported a Grant-in-aid for Scientific Research from the Japan Society for the Promotion of Science (No. 20592153) and a Grant for Supporting Project for Strategic Research of Nihon University School of Dentistry at Matsudo by the Ministry of Education, Culture, Sports, Science and Technology, 2008-2012.

\section{Refferences}

1. Jernvall $\mathrm{J}$ and Thesleff I. Reiterative signaling and patterning during mammalian tooth morphogenesis. Mech Dev 92: 1929, 2000

2. Thesleff I. Epithelial-mesenchymal signaling regulating tooth morphogenesis. J Cell Sci 116: 1647-1648, 2003

3. Maas R and Bei M. The genetic control of early tooth development. Crit Rev Oral Biol Med 8: 4-39, 1997

4. Kollar EJ and Baird GR. The influence of the dental papilla in the development of tooth shape in embryonic mouse tooth germ. J Embryo Exp Morphol 21: 131-148, 1969

5. Kollar EJ and Baird G.R. Tissue interaction in embryonic mouse tooth germ. II. The inductive role of dental papillae. J Embryo Exp Morphol 24: 173-186, 1970

6. Mina M and Kollar EJ. The introduction of odontogenesis in nondental mensenchyme combined with early murine mandibular arch epithelium. Arch Oral Biol 32: 123-127, 1987

7. Jiang TX, Jung HS, Widelitz RB and Chuong CM. Selforganization of periodic patterns by dissociated feather mesenchymal cells and the regulation of size, number and 
Hitoshi Yamamoto et al.: Periodontium by Reaggregation System

spacing of primordial. Development 126: 4997-5009, 1999

8. Yamamoto H, Kim EJ, Cho SW and Jung HS. Analysis of tooth formation by reaggregated dental mesenchyme from mouse embryo. J Electr Microscop 52: 559-566, 2003

9. Nakao K, Morita R, Saji Y, Ishida K, Tomita Y, Ogawa M, Saitoh M, Tomooka Y and Tsuji T. The development of a bioengineered organ germ method. Nature Methods 4: 227230, 2007

10. Cichon JC and Pack RS. Taurodontism: review of literature and report of case. J Am Dent Assoc 111: 453-455, 1985

11. Hamner JE, Witkop CJ and Metro PS. Taurodontism. Report of a case. Oral Surg 18: 409, 1946

12. Jorgenson RJ, Salinas, CF and Shapiro SD. The prevalence of taurodontism in a select population. J Craniofac Genet Dev Biol 2: 125-135, 1982

13. Higuchi Y, Yamamoto H, Cai J, Suzuki K, Jung HS and Kozawa Y (2008) Comparison of the structures of tooth germs transplanted into the subcutaneous tissue or the kidney capsule of mice. Int J Oral-Med Sci 7: 27-34, 2008

14. Hu B, Nadiri A, Kuchler-Bopp S, Perrin-Schmitt F, Peters H and Lesot $\mathrm{H}$. Tissue engineering of tooth crown, root, and periodontium. Tissue Eng 12: 2069-2075, 2006 
J.Hard Tissue Biology Vol. 18(2):77-82, 2009 